\title{
In situ detection of enterocytic apoptosis in normal colonic mucosa and in familial adenomatous polyposis
}

\author{
J Sträter, K Koretz, A R Günthert, P Möller
}

\begin{abstract}
Physiological regeneration of colonic epithelium entails proliferation at the crypt base and cell loss by shedding or cell death. The aim of this study was to localise and assess the rate of apoptosis in normal and neoplastic colonic epithelium with respect to zones of proliferation. Familial adenomatous polyposis (FAP) was chosen as a model to study neoplastic transformation of colonic mucosa at an early stage. Apoptotic cells were detected in situ by TdT-mediated biotin-dUTP nick end labelling (TUNEL) in parallel to cells in cycle determined by Ki-67 immunohistochemistry using the monoclonal antibody MiB-1. By detection of genomic fragmentation, two different patterns of enterocytic apoptosis emerged: (a) apoptotic bodies being engulfed by adjacent epithelial cells, and (b) apoptotic cells with only subtle morphological changes being extruded into the gut lumen. The engulfment pattern was seen predominantly in the crypts of the normal colonic mucosa and, although very rare, was clearly confined to the basal proliferation compartment. The extrusion pattern was restricted to the luminal mucosal surface. Adenomas of FAP showed highly increased numbers of apoptotic bodies, which were scattered throughout the transformed mucosa. Both patterns of apoptosis were topographically intermingled although the extrusion pattern prevailed at the luminal adenoma surfaces. Whereas cells in cycle were somewhat more numerous in the upper parts of the crypts, apoptosis occurred with increased frequency at sites beneath the proliferation maximum suggesting an inverted direction of epithelial cell migration in adenomas. These results suggest two distinct routes towards enterocytic apoptosis in the colonic mucosa leading to engulfment or extrusion of dying cells. Adenomatous transformation of colon epithelium is associated with a considerable increase of the cellular turnover rate and with a severe disturbance of the microtopographical localisation of birth and death of enterocytes.

(Gut 1995; 37: 819-825)
\end{abstract}

Keywords: apoptosis, colon, mucosa, adenoma, adenomatosis coli.

Correspondence to: Pathology, University of

Heidelberg, Im Neuenheimer

Feld 220, D-69120

Heidelberg, Germany.

Accepted for publication

11 May 1995

The process of renewal of the colonic mucosa entails enterocytic proliferation within the lower parts of the crypts, differentiation and migration along the crypt axis towards the surface epithelium, and, finally, cell loss by shedding at the luminal surface or intraepithelial cell death. ${ }^{1}$ In recent years, many studies have concentrated on proliferation kinetics and distribution of proliferating cells in the normal and neoplastic mucosa. ${ }^{2-11}$ Thus, the cell cycle time in colon and rectum was determined to last about one to two days ${ }^{12}$ while the total turnover time in the human colon has been estimated to range between three and eight days. ${ }^{12-14}$ Less, however, is known about mechanisms of cell loss in the intestine. It has been shown that cells of the luminal surface epithelium exhibit fragmentation of their DNA, ${ }^{15-17}$ suggesting that programmed cell death, apoptosis, is involved in the superficial loss of intestinal cells. Apoptosis is an active, irreversible process leading to progressive genomic fragmentation and structural degradation of the cell, which is complete after less than four hours. ${ }^{18}$ The aim of our study was to investigate more deeply apoptosis in the normal as well as in the neoplastic colonic mucosa with respect to the position of apoptotic cells within the mucosa and their relation to zones of proliferation. For this purpose, the recently described TdT-mediated biotin dUTP nick end labelling (TUNEL) method for the in situ detection of apoptotic cells ${ }^{15}$ was applied together with Ki-67 immunohistochemistry. Familial adenomatous polyposis (FAP) was chosen as a model to study neoplastic transformation of the colonic mucosa at an early stage characterised by limited structural complexity.

\section{Methods}

Tissue and tissue preparation

Tissue taken from colonic biopsy specimens or resection specimens was sent to our institute for routine diagnostics. Specimens were fixed in $6 \%$ formalin, routinely processed, and embedded in paraffin wax. We examined 16 colonic tissue samples from patients with FAP including non-neoplastic and adenomatously transformed mucosa. Adenomas examined ranged from macroscopically invisible, up to a size of $0.5 \mathrm{~cm}$ in diameter. All were of the tubular type. Normal colonic mucosa from 14 tumour free resection margins from colonic specimens resected because of sporadic cancer served as controls. Sections of about $4 \mu \mathrm{m}$ in thickness were adhered to glass slides that had been coated with $2 \%$ 3-aminopropyltriethoxy- 
Deparaffinisation was done by xylene, followed by rehydration in ethanol/water baths of falling ethanol concentration. Subsequently, deactivation of endogenous peroxidase in methanol/ $\mathrm{H}_{2} \mathrm{O}_{2}$ for 20 minutes was carried out. As we have shown that short-term microwave irradiation sensitises the TUNEL method for in situ detection of apoptotic cells, ${ }^{16}$ tissue sections were transferred to a $0.01 \mathrm{M}$ citrate buffer $(\mathrm{pH} 6.0)$ and treated in a microwave oven (Sharp, Hamburg, Germany) at $750 \mathrm{~W}$ before both the nick end labelling procedure and immunohistochemistry. Treatment duration was about three to five minutes for TUNEL and 15 minutes for immunohistochemistry.

\section{Nick end labelling procedure}

For the in situ detection of apoptotic cells, a modification of the TUNEL method described by Gavrieli ${ }^{15}$ was applied. Briefly, after digestion of nuclear proteins by proteinase $K$ (Merck, Darmstadt, Germany) for about 15 minutes, terminal deoxyribonucleotidyl transferase (TdT) (Promega, Madison, WI, USA) at a final concentration of $0 \cdot 1 \mathrm{EU} / \mu \mathrm{l}$ was used to insert Biotin-16-dUTP (Boehringer, Mannheim, Germany) to the ends of the DNA fragments. A streptavidin-peroxidase complex (Amersham, High Wycombe, UK) and 3amino-9-ethylcarbazole (AEC) (Sigma) served as a detection system for biotin, the peroxidase reaction resulting in an intense red precipitate. Finally, the sections were faintly counterstained with Harris haematoxylin. Negative controls were performed by omission of the transferase. This procedure did not show any non-specific reaction. Positive controls were done by DNAse I (Sigma) digestion of the DNA, giving rise to a strong nuclear staining of all cells. Apoptotic bodies within germinal centres of the Payer's plaques and in the interstitium served as additional intrinsic positive controls.

\section{Evaluation of apoptotic cell death}

In the untransformed mucosa, at least 12 well oriented crypts of each tissue sample were examined for apoptotic cells. Evaluation was restricted to those crypts whose entire length could be analysed. To determine the occurrence of apoptosis with respect to the cell position along the crypt axis, normal colonic crypts were longitudinally divided into five compartments from the base (compartment 1) to the luminal surface (compartment 5) ${ }^{19}$ Adenomas differed considerably in size and their tubules were often contorted, so that a division into the above defined compartments was inadequate. Thus, they were functionally divided into a morphologically non-neoplastic base (if any), composed of a proliferation zone (as defined by $\mathrm{Ki}$-67-expression, see later) (compartment I) and a differentiation zone (compartment II), and the more superficially located neoplastic mucosa, divided into three compartments of equal length from the basal parts of the neoplastic mucosa (compartment III) to the luminal surface (compartment V). Again, at least 12 adenomatous tubules were examined giving preference to those that were cut most longitudinally.

A preliminary evaluation showed some degree of interindividual and intraindividual variability in frequency of apoptotic events. Therefore, the frequency of apoptotic cells detected in a single compartment was assessed in a semiquantitative manner. The following semiquantitative score was set up: ' 0 ' absence of labelled cells; ' 1 ' single apoptotic cells in very few corresponding compartments of a larger mucosal area; ' 2 ' approximately one stained cell or microbody within the corresponding compartment of almost each crypt per specimen; ' 3 ' distinctly more than one apoptotic cell per compartment separated by gap-like 'apoptosis-free' regions; '4' high frequency of apoptotic bodies, lining the basal membrane with as few as one to five viable (unlabelled) enterocytes in between. In the case of marked heterogeneity within corresponding compartments of the same tissue section, both categories concerned were indicated (for example, $1 / 2 ; 2 / 3 ; 3 / 4$ ).

\section{Immunohistochemistry}

To relate the location of apoptosis with zones of proliferation, cells in cycle were immunohistochemically stained using an adjacent tissue section and applying the mouse monoclonal antibody MiB-1 (Dako, Copenhagen, Denmark), which recognised the $\mathrm{Ki}-67$ antigen in paraffin wax embedded tissue sections. ${ }^{20}$ The immunohistochemical staining method is described in detail elsewhere. ${ }^{21} \mathrm{~A}$ polyclonal biotinylated sheep antibody to mouse Ig (Amersham, High Wycombe, UK), the streptavidin-peroxidase complex (see above), and AEC served as a detection system for the primary antibody. Positive cells showed a clear cut nuclear signal. Negative controls were performed without the primary antibody. No non-specific staining was seen in these cases.

\section{Results}

Detection of genomic fragmentation by TUNEL resulted in an intense nuclear staining of apoptotic cells and apoptotic bodies. Both in the normal and neoplastically transformed colonic mucosa, two distinct patterns of apoptotic cell death emerged (Fig 1): (a) intraepithelial enterocytic apoptosis and subsequent engulfment of apoptotic bodies by adjacent epithelial cells (Fig 1A, C), and (b) apoptosis along with extrusion of enterocytes. These apoptotic cells show only very early morphological changes while, after having been extruded, they undergo progressive structural desintegration within the luminal mucus layer (Fig 1B).

\section{Normal colon epithelium}

Figure 2A summarises the findings in the normal colon epithelium. Apoptotic cells or 


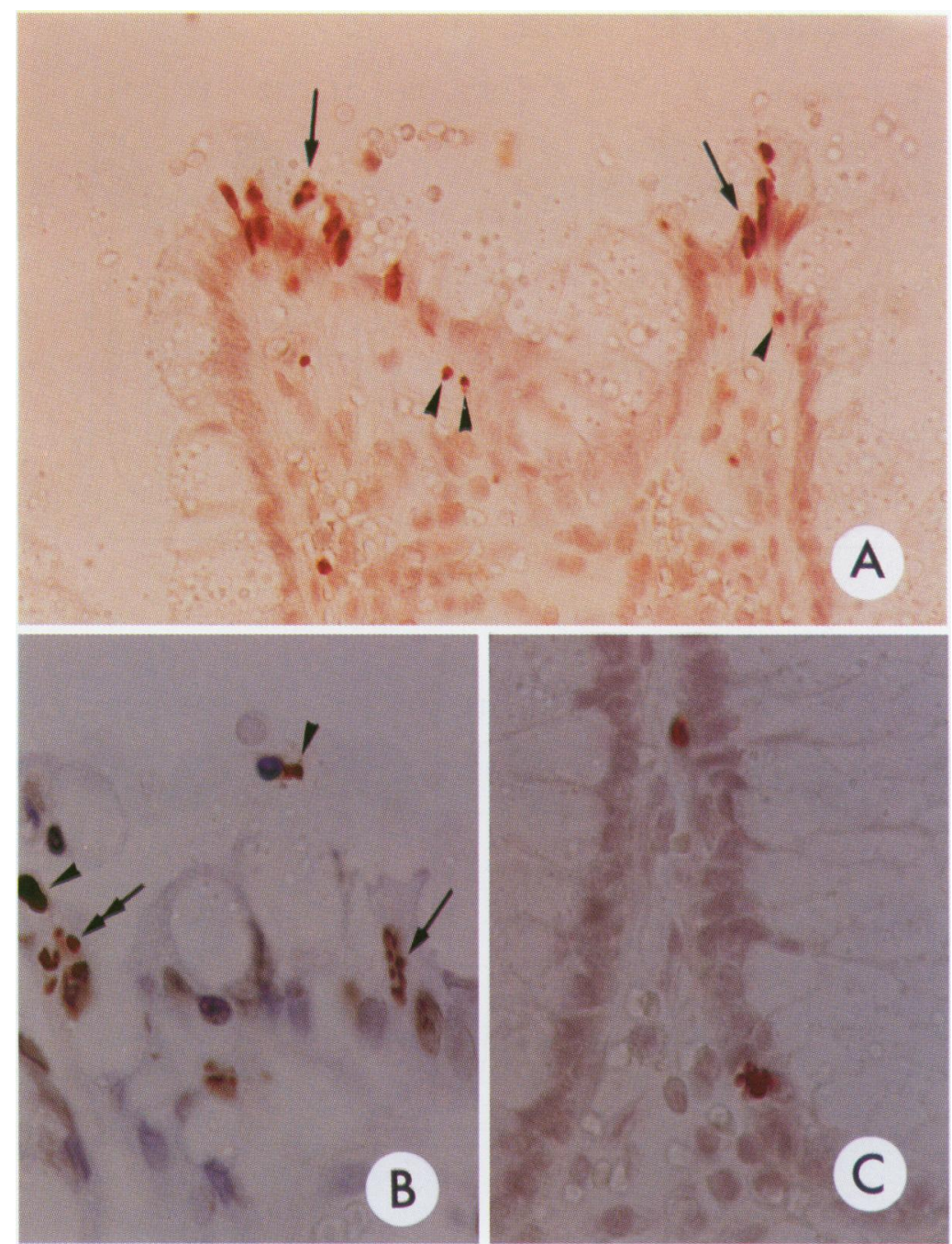

Figure 1: Two distinct patterns of apoptotic cell death are highlighted by TUNEL staining of the normal colon mucosa. (A) Cells being extruded into the gut lumen at early stages of apoptosis were more frequent than typical apoptotic bodies engulfed by adjacent viable cells (arrow heads). Note nuclear changes of cells while being extruded (arrows). Additionally, single apoptotic bodies in the lamina propria are labelled. Original magnification $\times 400$. (B) High power view of stained cells being extruded into the gut lumen ('extrusion pattern'). Note chromatin condensation at the nuclear periphery as early sign of apoptosis (arrow) and subsequent nuclear fragmentation (double arrow). Within the gut lumen, pycnotic nuclei are labelled (arrow heads) $\times 630$. (C) High power view of typical apoptotic bodies within the crypt base ('engulfment pattern') $\times 630$.

bodies were located within two different zones (Fig 3), the first of which comprised the lower parts of the crypts (compartments 1 and 2). Compartments 1 and 2 neatly corresponded to the proliferation zones as shown by detection of the Ki-67 antigen. Within these compartments, apoptotic cell death was a rather rare event with a mean frequency of much less than one apoptotic cell per crypt and always gave rise to the engulfment pattern (Fig 1C). In three of 14 tissue samples, apoptotic bodies were detected in these compartments at a slightly higher incidence of about one or two events per crypt.

The second and main region to contain considerable numbers of apoptotic cells or bodies was the luminal surface mucosa (compartment 5). In this compartment, labelled apoptotic figures varied considerably in number comprising single scattered cells, ranging up to most of the cells in at least some areas of the surface epithelium. Cell death giving rise to the engulfment as well as to the extrusion pattern was seen, however, in most cases, the extrusion type was more frequent (Fig 1A).
Apoptosis in the middle and upper parts of the crypts (compartments 3 and 4) was detected in single crypts in as few as two of 14 samples of normal colonic mucosa and, thus, was extremely infrequent.

\section{Untransformed mucosa in FAP}

Figure 2B summarises the data obtained from the untransformed mucosa in FAP. Even though minor morphological differences between the normal colonic mucosa and the untransformed mucosa in FAP were obvious (for example, extensive production of mucus with dilatation of the globlet cells and of crypt lumina in FAP), the frequency and distribution of apoptotic cells in these glands was essentially similar: labelled apoptotic cells or bodies were located mainly at the lower parts of the crypts and in the surface epithelium. Within compartments 1 and 2, again corresponding to the proliferation zone as characterised by $\mathrm{Ki}-67$ antigen expression, only single apoptotic bodies in very few crypts were seen. This was true for all cases but one, which did not show any apoptosis in these basal parts of the crypts. In four of 16 cases, apoptotic cell death was found occasionally in compartment 3 , whereas dying cells were absent in compartment 4. Similar to the normal state, apoptotic cell death within these lower compartments led to engulfment. The superficial epithelium (compartment 5) again showed significantly more labelled cells undergoing both engulfment and extrusion type of apoptosis but the extrusion pattern prevailed in most cases.

\section{Adenomas in FAP}

Figure 2C shows the results obtained from adenomas in FAP. In most small adenomas, especially in those of the oligotubular type, crypts were often composed of a morphologically normal base with neoplastically transformed epithelium on top. In the untransformed crypt base, apoptosis and engulfment were found again in small numbers within the proliferation zone as determined by $\mathrm{Ki}-67$ expression (compartment I). In compartment II, apoptotic cells were seen in just two cases, each of which contained very few nuclear fragments. Thus, distribution and frequency of apoptosis at the adenoma base resembled that seen in the crypts of the normal colonic mucosa. However, a peculiar feature (not shown in Fig 2C) was sometimes seen: close to the border of the neoplastic mucosa there was a sharp increase in apoptotic events all of them giving rise to the engulfment pattern (Fig 4).

Frequency and distribution of proliferating and dying cells were very different in the neoplastic part of the adenomas (Fig 5): (a) proliferation and apoptotic rates were highly increased, and (b) the distribution of proliferating cells and apoptotic events was highly abnormal. Cells in cycle were found most frequently in the upper parts of the neoplastic glands close to the luminal adenoma (compartment IV). However, apoptotic cells, in contrast, were found most frequently close to the 

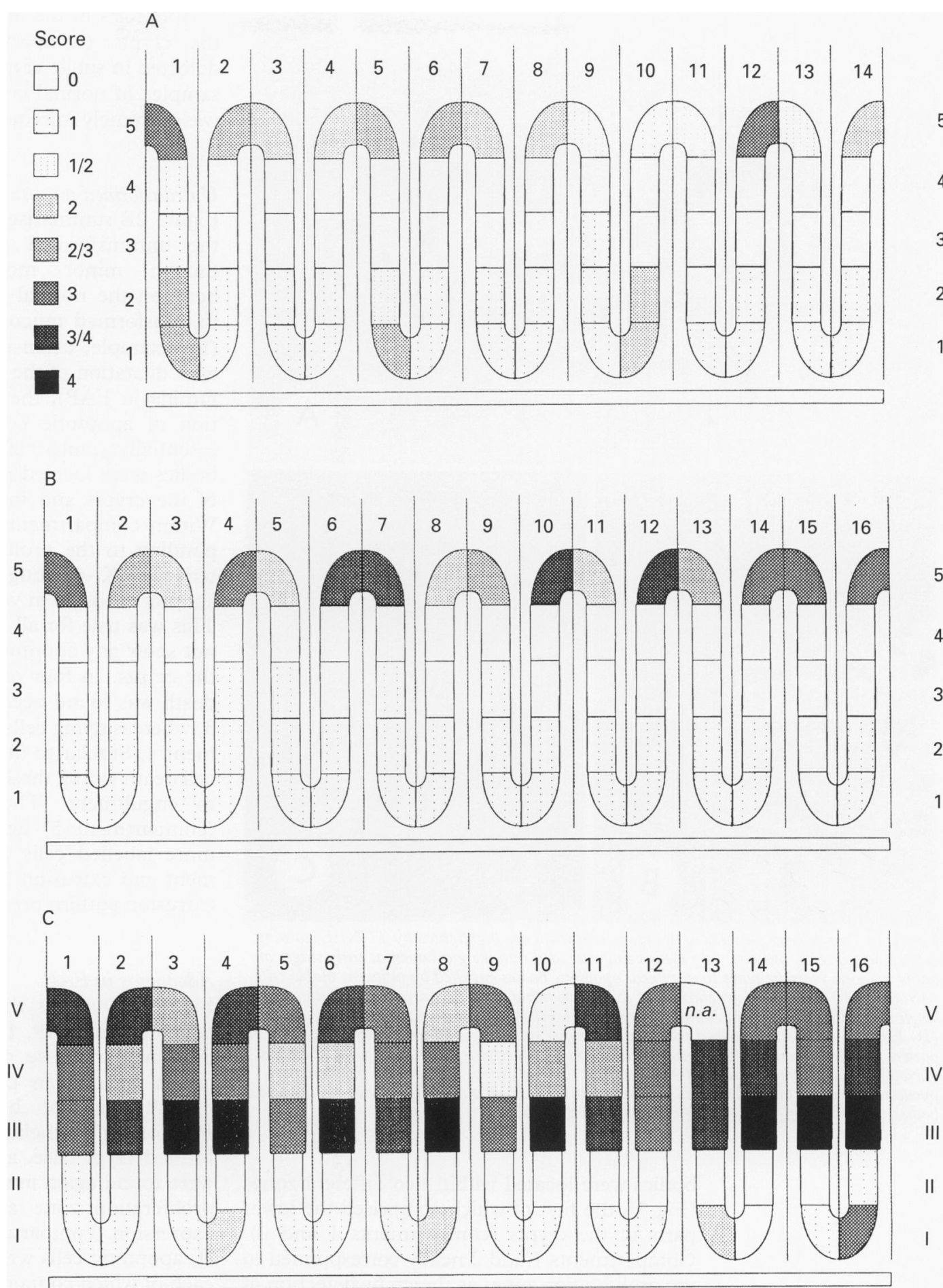

Figure 2: Frequency and distribution of apoptotic cells in the normal colon mucosa $(A)$, in untransformed mucosa in FAP $(B)$, and in adenomas in FAP (C). Crypts of the untransformed (Arabic numerals) and neoplastic (Roman numerals) mucosa were longitudinally divided into five compartments (see Methods). The compartment number is shown on the left and on the right hand side of the diagram. Each half-crypt represents one case (tissue sample) with at least 12 crypts examined, the case number being shown above. To show the number of apoptotic cells per crypt, a semiquantitative score was set up; the digit ' 0 '- '4' signify different frequencies within a certain crypt compartment with ' 0 ' representing the lowest and ' 4 ' the highest number of apoptotic cells or bodies (for details see Methods).

adenoma base - that is, close to the border of the underlying untransformed glands (compartment III). Thus, with respect to normal status, there was some sort of upside-down situation within the neoplastic part of the adenoma. Both patterns of apoptotic enterocyte elimination were present. Irrespective of the inverted relative frequencies of apoptotic events, the patterns of elimination corresponded to that of the normal situation (Fig 5): the extrusion pattern prevailed at the adenoma surface (compartment $\mathrm{V}$ ), while the engulf- ment pattern predominated along the neoplastic glands (compartments IV and III) (Fig 6).

\section{Discussion}

We have shown that in normal colonic mucosa, apoptotic death of enterocytes leads to two different patterns of elimination, (a) engulfment by adjacent enterocytes and (b) extrusion. In the normal state these patterns are confined to distinct micro-environments. Labelled apoptotic cells giving rise to the 


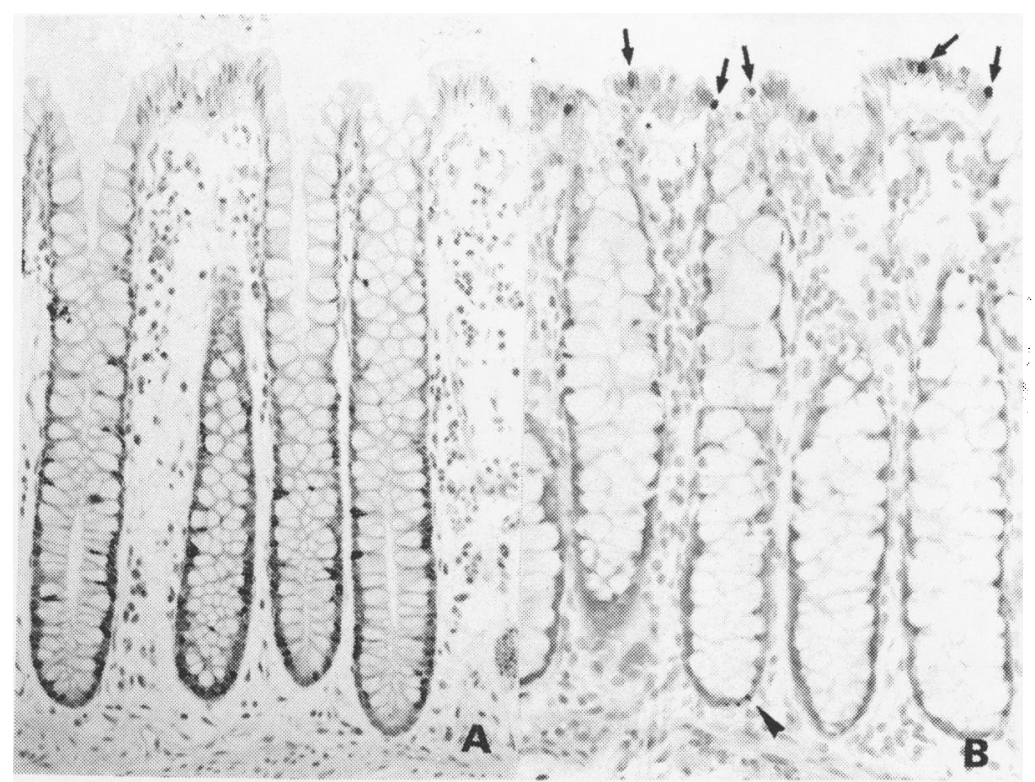

Figure 3: Normal colon mucosa; original magnification $\times 200$. (A) Typical immunohistochemical staining of cells in cycle (at the crypt bases) by the monoclonal antibody MiB-1 recognising the Ki-67-antigen. (B) Detection of DNA fragmentation using the TUNEL technique. Labelled cells are seen in two different compartments: single apoptotic bodies are detected within the proliferation compartment at the base of some crypts (arrow head); more frequently, however, cells exhibiting DNA fragmentation are visualised within the surface epithelium (arrows).

engulfment pattern were located within the proliferation zones located at the bottom of the crypts, whereas the extrusion pattern was predominate at the luminal surface. These findings confirm and extend published data.

Already in the 1950s, cytoplasmic inclusion bodies containing DNA had been seen in human rectal polyps by Leuchtenberger, ${ }^{22}$

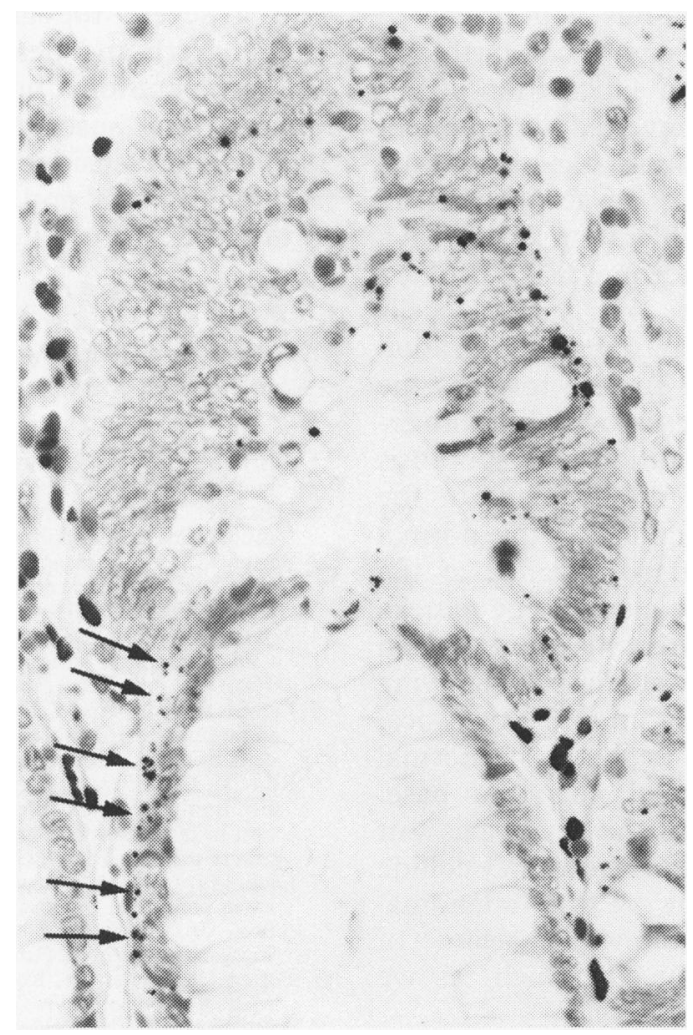

Figure 4: Border area of untransformed mucosa (bottom) and adenoma in FAP (top). Several apoptotic bodies are detected within the transformed mucosa. Additionally, apoptotic remnants are seen lining the basal membrane of the untransformed mucosa most adjacent to the adenoma (arrows). Original magnification $\times 400$. and, recently, the occurrence of 'Leuchtenberger bodies' was described to be a characteristic of adenomas in FAP. ${ }^{23}$ In both reports, these bodies have been misinterpreted as being either viral inclusion bodies or remnants of intraepithelial lymphocytes. Our finding that these bodies contain high amounts of DNA fragments and regularly occur within the proliferative basal zone of normal glands shows strong evidence that these are apoptotic bodies. The significance of spontaneous apoptotic cell death in the proliferation compartment is unknown. It has been speculated that spontaneous apoptosis in the basal parts of the crypts may result from an occasional symmetrical stem cell division in a system that normally divides asymmetrically ${ }^{24}$ to prevent hyperplasia. After irradiation and after administration of cytotoxic drugs, apoptotic bodies are seen in dose dependent numbers predominantly in the basal proliferation zone of colonic crypts. ${ }^{25-27}$ This, however, suggests that enterocytes die by apoptosis whenever mitosis fails.

Using the TUNEL technique, Gavrieli et al ${ }^{15}$ showed DNA breaks in epithelial cells located in the villus tips in the rodent and human small intestine, suggesting involvement of apoptosis in the process of physiological gut mucosa regeneration. These data were supported by Ansari et al ${ }^{17}$ who applied an in situ nick translation technique. However, these authors refrained from accepting these labelled cells as being apoptotic because of the lack of clear cytological criteria of programmed cell death. They speculated that DNA breaks might be secondarily caused by artefacts of fixation and tissue processing. Nevertheless, ultrastructural studies by Potten and Allen ${ }^{28}$ showed that, close to the small intestinal villus tips, apoptosis actually occurs and apoptotic cells may undergo extrusion into the gut lumen rather than getting engulfed by adjacent cells. Considering these and our own findings, it is at least possible that one route towards apoptosis starts by detachment from the basal lamina, which provides necessary survival signals. This would be in accord with a recent report on apoptosis induction by disruption of epithelial cell/matrix interaction, a phenomenon called 'anoikis' by the authors. ${ }^{29}$ On the other hand, it is known that apoptotic cells tend to rapidly detach from adjacent structures. ${ }^{30}$ In our case, it is unclear which of the two phenomena is the cause and which is the consequence. The 'anoikis' concept, however, apparently does not apply to the engulfment pattern of enterocyte elimination, which is the consequence of apoptosis in situ. This suggests that enterocytes might have a second route at apoptosis. Alternatively, there might be one apoptotic programme that can be modulated by factors/signals differing microtopographically along the maturation pathway of the enterocyte. Our comparative analysis of frequencies of cells in cycle and cells undergoing apoptosis showed that, in the neoplastic part of adenomas, both processes are considerably increased thus illustrating an abnormally high turnover rate. Cycling cells were more frequent 


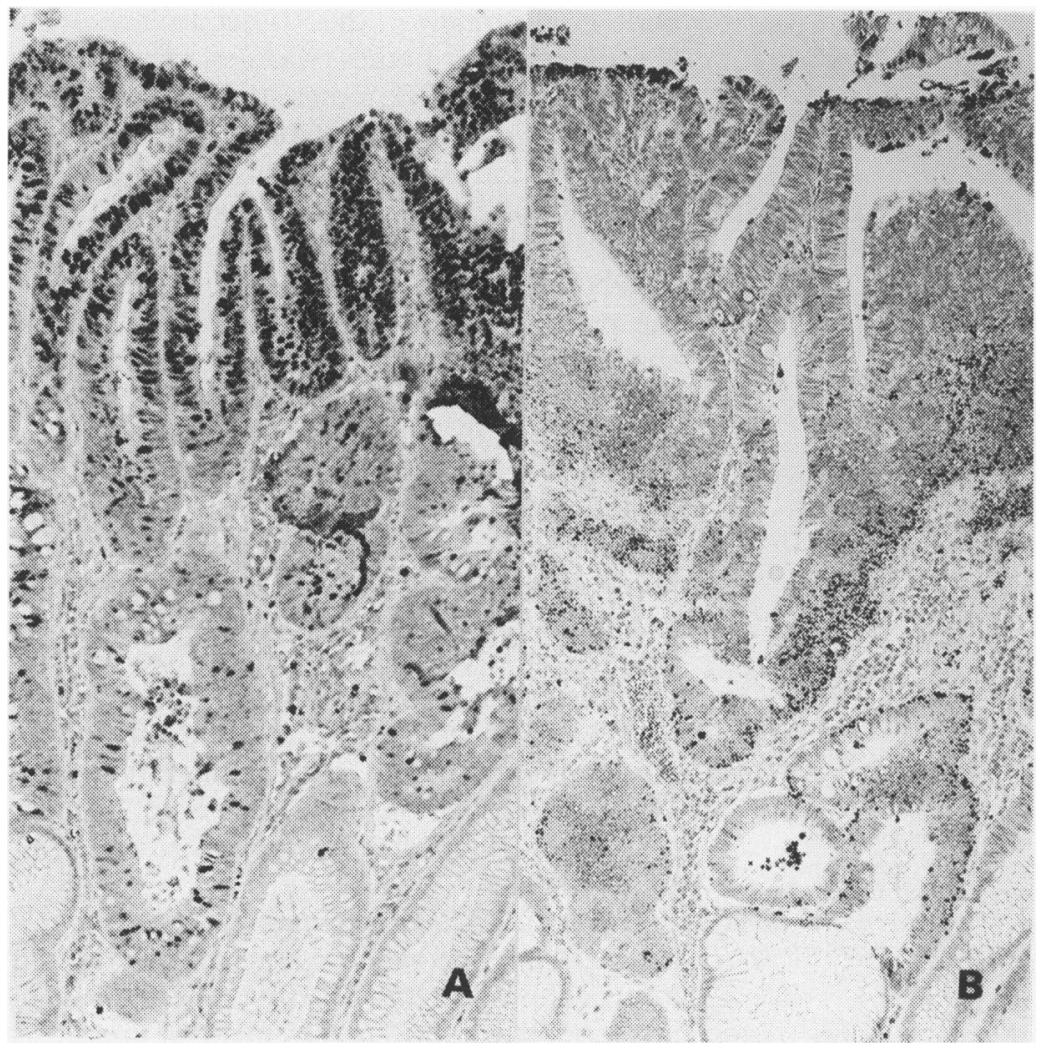

Figure 5: Serial paraffin wax sections of an adenoma in FAP; original magnification $\times 100$. (A) Immunohistochemical staining of Ki-67-positive cells. Note that stained cells are predominantly in the superficial compartments with only few Ki-67-positive cells in the more basal parts of the crypt. (B) Detection of DNA fragmentation by TUNEL. Apoptotic bodies are scattered throughout the transformed mucosa, most often lining the basal membrane, the maximum amount of bodies found within deeper parts of the crypts. Note that within the surface epithelium, the pattern of apoptotic cells being extruded is maintained.

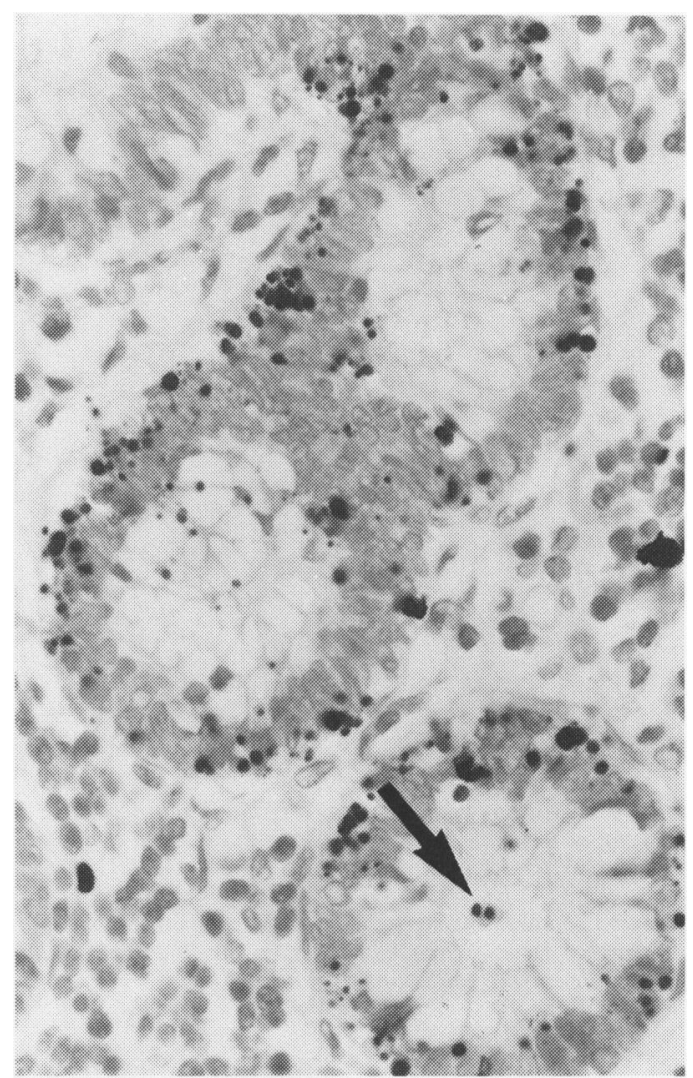

Figure 6: Transverse section through adenoma crypts in FAP. Numerous apoptotic remnants are detected within the epithelium most of them lining the basal lamina. Only single apoptotic bodies were extruded into the crypt lumen (arrow). Original magnification $\times 400$.

extrusion. Adenomatous transformation of the colonic epithelium in FAP is associated with an increased turnover rate of neoplastic cells and with a severe topographical disturbance of cellular birth and death. Despite deregulated cell proliferation, however, adenoma cells remain either susceptible to signals inducing apoptosis or sensitive to apoptosis by growth factor withdrawal and thus remain under some sort of 'social control'.

This study was supported by grants from the Deutsche Krebshilfe (W $8 / 92 / \mathrm{Kol}$ ) and the Tumorzentrum Heidelberg/Mannheim. We thank Mrs B Hein, Mrs A Müller, Mrs B Weigold, and Mrs S Westenfelder for excellent technical assistance and Mr Mark Meier for help with the manuscript. showed their abnormal retrograde migration away from the adenoma surface towards the crypt base. ${ }^{5}$ However, the topographical inversion of regions of proliferation and apoptosis did not affect the local confinement of the patterns of apoptosis. Similar to the normal state, the extrusion pattern prevailed at the adenoma surface and the engulfment pattern predominated at the adenoma base, again suggesting that the mode of eliminating dying enterocytes depends on modulating local factors. This would also apply to the normal cells of the untransformed adenoma base. These cells migrate along the crypt axis but cannot reach the luminal surface as they collide with the neoplastic part of the adenoma. Consequently, the engulfment pattern of apoptosis should be induced, which, as we show (Fig 4), is indeed the case.

Summarising, we conclude that, within the colonic mucosa, two distinct patterns of apoptotic enterocytic cell death exist, illustrated by two different modes of elimination of cells programmed for apoptosis, engulfment and
1 Eastwood GL. Progress in gastroenterology: Gastrointestinal epithelial renewal. Gastroenterology 1977; 72: 962-75.

2 Deschner EE, Lipkin M. Proliferative patterns in colonic mucosa in familial polyposis. Cancer 1975; 35: 413-8.

3 Potten CS. Kinetics and possible regulation of crypt cell populations under normal and stress conditions. Bull Cancer 1975; 62: 419-30.

4 Al-Dewachi HS, Wright NA, Appleton DR, Watson AJ. Cell population kinetics in the mouse jeju

5 Lightda $C$, $B$ Cell Pathol 1975; 18. 225-42. in familial polyposis: kinetics and location of proliferating cells in colonic adenomas. Cancer Res 1982; 42: 4280-3. FP. Immunohistochemical study of epithelial cell proliferation in hyperplastic polyps, adenomas, and adenocarcinomas of the large bowel. Gastroenterology 1988; 94: 899-906.

7 Diebold J, Lai MD, Löhrs U. Analysis of proliferative activity in colorectal mucosa by immunohistochemical detection of proliferating cell nuclear antigen (PCNA) Virchows Arch B Cell Pathol 1992; 62: 283-9.

8 Potten CS, Kellett M, Roberts SA, Rew DA, Wilson GD. Measurement of in vivo proliferation in human colorecta

9 Yoshikawa R, Hatada T, Kusunoki M, Yamamura T, Utsunomiya J. Assessment of cell proliferation kinetics in familial adenomatous polyposis by PCNA immunostaining. Int f Oncol 1993; 3: 341-5.

10 Roncucci L, Pedroni M, Rante R, Di Gregorio C, Ponz de Leon M. Cell kinetic evaluation of human colonic aberrant crypts. Cancer Res 1993; 53: 3726-9. mucosa using bromodeoxyuridine. Gut 1992; 33: 71-8. 
11 Deschner EE, Raicht RF. Kinetic and morphologic alterations in the colon of a patient with multiple polyposis. Cancer 1981; 47: 2440-5

12 Lipkin M, Sherlock P, Bell B. Cell proliferation kinetics in the gastrointestinal tract of man. II. Renewal in stomach, ileum, colon, and rectum. Gastroenterology 1963; 45: 721-9.

13 Cole JW, McKalen A. Observations of cell renewal in human rectal mucosa in vivo with thymidine-H3. human rectal mucosa in vivo

14 MacDonald WC, Trier JS, Everrett NB. Cell proliferation and migration in the stomach, duodenum, and rectum of man: radioautographic studies. Gastroenterology 1964; 46: 405-17.

15 Gavrieli Y, Sherman Y, Ben-Sasson SA. Identification of programmed cell death in situ via specific labeling of nuclear DNA fragmentation. $\mathcal{F}$ Cell Biol 1992; 119: 493-501.

16 Sträter J, Günthert AR, Brüderlein S, Möller P. Microwave irradiation of paraffin-embedded tissue sensitizes the TUNEL-method for in situ detection of apoptotic cells. Histochemistry 1995; 103: 157-60.

17 Ansari B, Coates PJ, Greenstein BD, Hall PA. In situ endlabelling detects DNA strand breaks in apoptosis and other physiological and pathological states. $\mathfrak{f}$ Pathol 1993; other physio: $1-8$.

18 Wyllie AH, Kerr JFR, Currie AR. Cell death: the significance of apoptosis. Int Rev Cytol 1980; 68: 251-306.

19 Lipkin M. Biomarkers of increased susceptibility gastrointestinal cancer: new application to studies of cancer prevention in human subjects. Cancer Res 1988; 48: 235-45.

20 Key G, Becker MH, Baron B, Duchrow M, Schlüter C, Flad HD, et al. New Ki-67-equivalent murine monoclonal antibodies (MiB 1-3) generated against bacterially expressed parts of the Ki-67 cDNA containing three 62 base pair repetitive elements encoding for the $\mathrm{Ki}-67$ epitope. Lab Invest 1993; 68: 629-36.

21 Mielke B, Möller P. Histomorphologic and immunophenotypic spectrum of primary gastrointestinal B-cell lymphomas. Int $\mathcal{f}$ Cancer 1991; 47: 334-43.

22 Leuchtenberger C. Cytoplasmic 'inclusion bodies' containing desoxyribose nucleic acid (DNA) in cells of human rectal polyps. Lab Invest 1954; 3: 132-42.

23 Rubio CA, Alm T, Aly A, Poppen B. Intraepithelial bodies in colorectal adenomas: Leuchtenberger bodies revisited. in colorectal adenomas: Leuchtenb

24 Loeffer M, Birke A, Winton D, Potten CS. Somatic mutation, monoclonality and stochastic models of stem cell organisation in the intestinal crypt. $\mathcal{F}$ Theor Biol 1993; 160: 471-91.

25 Potten CS. Extreme sensitivity of some intestinal crypt cells to $X$ and $g$ irradiation. Nature $1977 ; 269: 518-21$.

26 Ijiri K, Potten CS. Further studies on the response of intestinal crypt cells of different hierarchical status to eighteen different cytotoxic agents. Br $\mathcal{f}$ Cancer 1987; 55: 113-23.

27 Potten CS, Li YQ O'Connor PJ, Winton DJ. A possible explanation for the different cancer incidence in the intestine, based on distribution of the cytotoxic effects of cartine, based on distribution of the cytotoxic effects of car-
cinogens in the murine large bowel. Carcinogenesis 1992; cinogens in the

28 Potten CS, Allen TD. Ultrastructure of cell loss in intestinal mucosa. F Ultrastructure Res 1977; 60: 272-7.

29 Frisch SM, Francis H. Disruption of epithelial cell-matrix interactions induces apoptosis. F Cell Biol 1994; 124: 619-26.

30 Searle J, Kerr JFR, Bishop CJ. Necrosis and apoptosis: distinct modes of cell death with fundamentally different signification. F Pathol Annu 1982; 17: 229-59. 